\title{
A Practical Method of Optimal RSUs Deployment for Smart Internet of Vehicle
}

\author{
Honghan Zhang* \\ ${ }^{*}$ Harbin Engineering University, Harbin, P. R. China \\ Email: oceanzhh@hrbeu.edu.cn
}

\begin{abstract}
The roadside units (RSUs) are absolutely indispensable elements in sparse highway senario for smart Internet of Vehicle (IoV). Most recent RSU deployment methods consider vehicle mobility, warning message reception probability and time delay respectively, do not mentioned the situation once the accident vehicle cant send the warning message. In this paper, we present a comprehensive analysis that integrates all these factors above. In particular, we model three kinds of common highway scenarios and give the closed-form expression of RSUs deployment number along the highway. The proposed method has been validated by extensive simulations using Matlab and NS2, its performance has been compared with TAPC method. Results reveal that our proposed method has better performance under the condition of high warning message probability.
\end{abstract}

Index Terms-Smart IoV, RSU deployment, time delay, reception probability, mobility.

\section{INTRODUCTION}

\section{A. Background and Significance}

The Global Status Report on Road Safety 2013 shows that 1.24 million people were killed on the worlds roads in 2010. This is unacceptably high. Road traffic injuries take an enormous toll on individuals and communities as well as on national economies. Middle-income countries, which are motorizing rapidly, are the hardest hit [1]. Smart internet of vehicle (IoV) is a promising Intelligent Transportation System (ITS) technology, which enables vehicles to communicate with each other and Road Side Units (RSU) by wireless radio. Recently, smart IoV have rapidly developed due to its safety applications, such as emergency warning message propagation, rescue message dissemination [2].

The roadside units(RSU) are an absolutely indispensable element in sparse highway for smart IoV, because Vehicleto-Roadside(V2R) communication is essential, as Vehicle-toVehicle(V2V) communication is not enough to support safety message propagation only. Meanwhile vehicles nearby driving in the same direction and at almost the same velocities can group a cluster to reduce the communication blind areas [2][6]. Distinct from Urban Scenario, the traffic flow density is low in Highway Scenario. Once an accident happens in the area where there are no vehicles within its communication range or an accident is severe, the communication devices on the vehicles are damaged. The following vehicles could cost more time to take notice, which increases the probability of a second accident, and furthermore it may cause traffic jam. But the cost may be high to cover whole area, and relatively the message time delay will be large if are deployed only a few RSUs. A rational number of RSUs along the highway is necessary. The problem of RSU deployment along the highway can be thought of from two aspects:

(1)What is the least number of RSUs that should be deployed while smart IoV can provide a certain level of connectivity and Quality of Service(QoS) for safety applications?

(2)What is the best RSU distribution along the roads that satisfies the safety message propagation as well as emerging the alert message?

We consider that RSU deployment should be uniformly distributed between two hot spots along the highway, because the vehicle and the accident site distribution is a stochastic process. In this respect, our contribution in this paper is presenting a RSU generating warning message method. Then we propose a new RSUs deployment method, that considers a wide range of scenarios unlike the other related works, which consider only a few aspects such as RSU coverage radius from technical view or the traffic flow density from traffic view. In this paper, we concentrate on the delay analysis for emergency message propagation both in the single vehicle and vehicle in the cluster scenario. We only calculate the meaningful time delay that is useful for the vehicles approaching the accident site. Due to the long distance from the accident site, vehicles have enough time to respond to the accident including reducing speed or leaving from the nearby exit. Finally we give the closed-form expression of RSU numbers along the highway.

\section{B. Ralated Works}

As former work concerning RSUs deployment, Liu et al. consider delay analysis through the curve fitting method, and present a mathematical model and analysis for broadcasting delay in cluster based Vehicular Ad Hoc Networks (VANETs) [7]. However, the optimal results are equal to full coverage numbers, so that the cost of deployment is high. From the economic point of view, this is unacceptable. O Trullols et al. maximize the number of vehicles that get in contact with the RSUs over the considered area. They formulate the problem as a maximum coverage problem [8]. They find the knowledge of vehicular mobility is a main factor in achieving an optimal deployment, while our simulation results are obtained under given traffic flow densities. Baber Aslam et al. present an analytical Binary Integer Programming(BIP) method and a novel Balloon Expansion Heuristic(BEH) method for placement of a limited number of RSUs in an urban region, then after comparison finds that $\mathrm{BEH}$ is more versatile and performs 
better than BIP [9]. Both two methods need to use enumeration method, which becomes inefficient with the increase in the size of the region. Tao et al. study the vehicle mobility characteristics along the highway and propose a Cluster-based RSU deployment scheme with Traffic-Aware Power Control Method to maximize the network performance, as well as minimizing the energy consumption of RSUs [10]. However the model does not consider the situation without the clusters, while we take consideration of both with and without the cluster in our model. Cumbal et al. present a RSU deployment method for an ideal Multi-hop communication environment between vehicles and RSUs [11]. We think the safety message propagation is unreliable through multi-hop communication. Xiong et al. transform the gateway deployment problem into a vertex selection problem in a graph, and propose a heuristic algorithm to find the optimal places. The algorithm utilizes the fine-grained statistical characteristic of mobility [12].The starting point of the algorithm is trying to keep the connection between vehicles and RSUs, which we think is not necessary. Cheng et al. propose a genetic algorithm-based sparse coverage with statistical analysis. They model a resourceconstrained problem as an NP-hard budget coverage problem [13]. However the algorithm needs a lot of iterations to achieve the results. Filippini et al. study the dynamics of infrastructure deployment by using game theoretical tools [14], however, the model is not suitable for safety message propagation. Renzo Massobrio et al. introduce a multi-objective formulation of the problem of locating roadside infrastructure for vehicular networks over realistic urban areas. They consider the deployment cost, and a traffic and coverage model for quality-of-service is defined, but they do not take consideration of accidents as a more realistic scenario in the problem, which we define in this paper [15]. Ahmed Makkawi et al. introduce a cumulative weight based method as a solution to the placement problem in the urban, rural and mountainous areas. The method gets the weight of each site of interest and adds the weights of the surrounding neighbors to its weight and considers the highest weight first in the distribution process [16]. The problem of RSU placement is formulated to binary integer programming by Nik Mohammad Balouchzahi et al. They present an optimization method based on BIP to find optimal locations for RSU installation in highway and urban scenarios. However, the model does not consider the influence of the traffic densities [17]. Xu Liya et al. investigate the problem of optimal RSU placement by developing a randomized algorithm, which gives an approximation to the optimal distance to guarantee the information can be passed to RSUs from the accident site via the VANET. However, the algorithm ignores the speed distribution function, which we take consideration into our model [18]. Tsung-Jung Wu et al. propose a capacity maximization placement scheme. Although it adapts to different vehicle population distribution and different vehicle speeds on the road, when the vehicle distribution exhibits more fluctuations, the set of RSUs is spaced apart more uniformly on the road [19]. The scheme also does not consider the situation of accidents, which will fluctuate the vehicle distribution and make it less accurate. And it also does not give the closed-form expression of the
RSU numbers.

\section{Methods}

\section{A. Highway model}

Here we suppose all the vehicles are equipped with communication devices which support both V2V and V2I mode. Highways are usually segregated by toll stations or rest stops. Due to the convenience of connecting to other networks, RSU should be deployed on the toll stations or the rest stops. Therefore we only need to consider the road segment between two toll stations or rest stops. We suppose $L$ miles straight path between two stations, and the inter vehicle distances are i.i.d and exponentially distributed with parameter $\rho$. Specifically, the $\mathrm{CDF}$ of the inter-vehicle distance $x$ is given by

$$
F(x)=1-e^{-\rho x}, x \geq 0 .
$$

Vehicles nearby are apt to form clusters. Once the cluster is formed, we suppose the vehicle will maintain its speed for stable communications. Taking account of the performance of clusters, we assume that vehicles within the cluster communicate with each other no more than two-hops, so the cluster size can make a simple approximation by

$$
S_{\text {cluster }}=\phi R, 0 \leq \phi \leq 2,
$$

where $R$ denotes the maximum communication range. $R$ also satisfies the threshold of SNR, and $\phi$ is the cluster size expansion coefficient that is flexible and dependent on the communication hops. Suppose that the RSUs are uniformly distributed along the road segments, and there are N RSUs deployed between the stations. For the simplicity of analysis we assume the same communication range between $\mathrm{V} 2 \mathrm{~V}$ and V2I mode. The blind area distance between two adjacent RSUs is given by

$$
S_{b l i n d}=\frac{L}{N+1}-2 R
$$

Figure 1 shows the relationship among the parameters in (3).

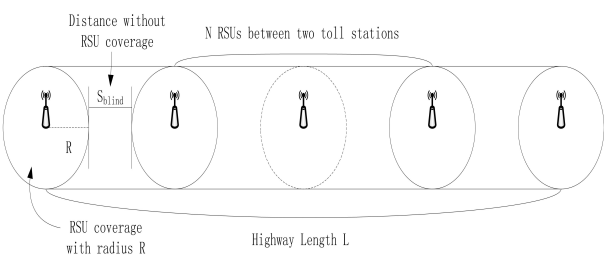

Fig. 1 RSU deployment in highway model.

When traffic flow density(TFD) is high, there will always be $\mathrm{V} 2 \mathrm{~V}$ communication link, so the time delay lower bound is equal to the radio transmission delay. However, when the TFD is low, the $\mathrm{V} 2 \mathrm{~V}$ communication link may be rare. if an accident happens out of the RSU coverage area that we defined as blind areas, the alerting message will not disseminate through IoV, or the accident happens in the RSU coverage areas, but it is severe so that the communication devices are damaged, the vehicle cannot make the alert message, RSUs will generate it like in Figure 2. 


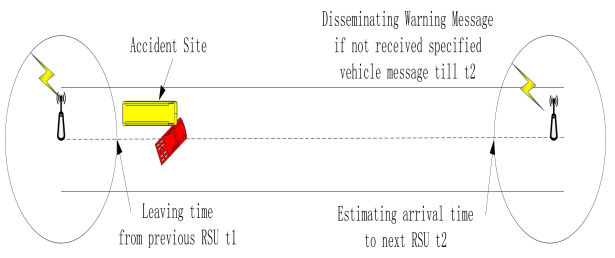

Fig. 2 Warning message jammed in highway model.

So the RSU generated warning message time delay is given by

$$
t_{\text {rsu }}=\frac{S_{\text {position }}}{v}, 0 \leq t \leq \frac{S_{\text {blind }}}{v_{\text {min }}}
$$

where $v$ enotes speed of the vehicle reported to the former RSU last time. So if the next RSU does not receives the heartbeat message after $t_{r s u}$. It will generate warning message and broadcast to the previous RSUs. We ignore the wireless propagation delay and the time delay between RSUs. The position where the accident happens obeys the uniform distribution is shown by

$$
f\left(S_{\text {position }}\right)=\frac{1}{S_{\text {blind }}}, 0 \leq S_{\text {position }} \leq S_{\text {blind }} .
$$

The probability density function of vehicle speed $v$ is a truncated Gaussian PDF given by

$$
f(v)=\frac{\frac{2}{\sigma_{v} \sqrt{2 \pi}} \exp ^{\left(\frac{-\left(v-\mu_{v}\right)^{2}}{2 \sigma_{v}^{2}}\right)}}{\operatorname{erf}\left(\frac{v_{\max }-\mu_{v}}{\sigma_{v} \sqrt{2}}\right)-\operatorname{erf}\left(\frac{v_{\min }-\mu_{v}}{\sigma_{v} \sqrt{2}}\right)}, v_{\min } \leq v \leq v_{\max }
$$

where $\mu_{v}$ is the average speed, $\sigma_{v}$ is the standard deviation of vehicle speed, the maximum speed $v_{\max }=\mu_{v}+3 \sigma_{v}$, the minimum speed $v_{\min }=\mu_{v}-3 \sigma_{v}$, erf $(\cdot)$ is the error function [20]. Due to the position and the speed are independent, the PDF of RSU time delay is given by

$$
f\left(t_{r s u}\right)=\int_{v_{\min }}^{v_{\max }} v \frac{f(v)}{S_{\text {blind }}} d v
$$

If the vehicle is located close to the accident site, it will has less time to decelerate or dodge. We only discuss the vehicles in the closest blind areas situation as Figure 3,4,5 described.

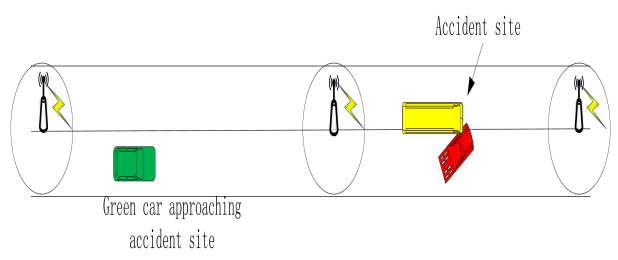

Fig. 3 Vehicle approaching accident site in highway model.

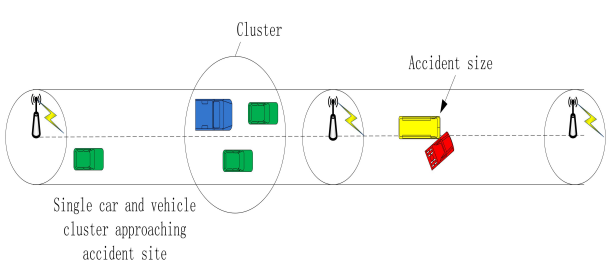

Fig. 4 Vehicle cluster approaching accident site in highway model.

Figure 3 illustrates the low TFD scenario. The vehicle at a moderate speed will obtain the warning message when

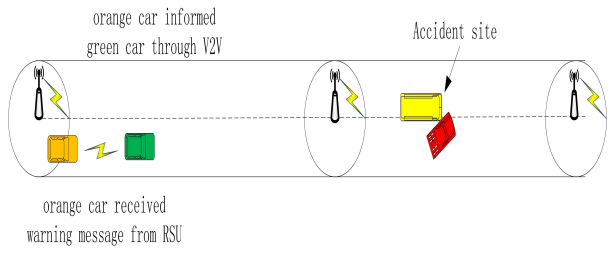

Fig. 5 Vehicle recieving the message from behind in highway model.

it comes into the RSU coverage area. Figure 4 illustrates the medium TFD scenario and there is a cluster where the vehicle will stay for some purpose like cooperative driving or infotainment sharing. Figure 5 shows another situation, in which the warning message is delivered by the vehicles behind. Here we do not consider the radio transmission delay and the data processing delay. Similar to other documents, the warning message is without feedback or retransmission. To avoid a secondary accident, the following vehicle needs a time advance. Let the minimum advance be $\Psi$. Here we simply assume $\Psi$ is the time that a vehicle decelerates its speed from $100 \mathrm{~km} / \mathrm{h}$ to $0 \mathrm{~km} / \mathrm{h}$, defined as $\Psi=15 \mathrm{~s}$. For the sake of simplicity, the minimum safety distance is $S_{\text {Safemin }}=\Psi v$.

We first calculate the vehicle time delay under situation in Figure 3. The larger the $t_{r s u}$, the higher the probability of the following vehicle passing RSU radio coverage without receiving an alerting message. If drivers only depend on visual observation, this is a loss of safety functionality of RSU deployment plan. The following vehicle position is $0 \leq$ $S_{\text {position }} \leq S_{\text {blind }}$. The CDF of $S_{\text {position }}$ is $\frac{S_{\text {blind }}}{S_{\text {blind }}+2 R} \sim 1$, as $S_{\text {blind }} \gg 2 R$. The distance of following vehicle after the accident happens is $0 \leq S_{\text {vehicle }} \leq v t_{\text {rsumax }}$. The distance of alerting is $0 \leq S_{\text {alert }} \leq S_{\text {blind }}+v t_{\text {rsumax }}$. In order to guarantee the following vehicle to have absolutely time advance, we need ensure $S_{\text {blind }}+2 R-S_{\text {alert }} \geq S_{\text {safemin }}$. That is $2 R-v t_{\text {rsumax }} \geq S_{\text {safemin }}$. Substituting $S_{\text {safemin }}$ we have

$$
\frac{2 R}{v}-t_{\text {rsumax }} \geq \Psi
$$

Another calculation of the vehicle time delay under the situation in Figure 5, which is also low traffic flow density situation. Suppose the yellow car receives a warning message through RSU broadcasting and it is faster than the green car. According to common human behaviors or the smart safety functionality, the yellow vehicle will now decelerate. We consider the situation that the green car keeps its speed, and receives warning message through $\mathrm{V} 2 \mathrm{~V}$ communication by the yellow car. The model is given by

$$
\begin{aligned}
& v_{1} t+S_{\text {position }}-v_{2} t \leq R \\
& \text { s.t. } v_{2}>v_{1}, R<S_{\text {position }}<S_{\text {blind }}, t \leq \Psi .
\end{aligned}
$$

Since the PDF of velocity is $f(v)$, the PDF of velocity 
difference is given by

$$
\begin{aligned}
& f\left(v_{d}\right)=\frac{g\left(\mu_{v_{1}}, \sigma_{v_{1}}\right) g\left(\mu_{v_{2}}, \sigma_{v_{2}}\right)}{\sqrt{2 \pi\left(\sigma_{v_{1}}^{2}+\sigma_{v_{2}}^{2}\right)}} \exp \left(\frac{-\left(v_{d}-\left(\mu_{v_{1}}-\mu_{v_{2}}\right)\right)^{2}}{2\left(\sigma_{v_{1}}^{2}+\sigma_{v_{2}}^{2}\right)}\right) \\
& g\left(\mu_{v_{1}}, \sigma_{v_{1}}\right)=\frac{2}{\operatorname{erf}\left(v_{\max }, \mu_{v_{1}}, \sigma_{v_{1}}\right)-\operatorname{erf}\left(v_{\min }, \mu_{v_{1}}, \sigma_{v_{1}}\right)}(12) \\
& g\left(\mu_{v_{2}}, \sigma_{v_{2}}\right)=\frac{2}{\operatorname{erf}\left(v_{\max }, \mu_{v_{2}}, \sigma_{v_{2}}\right)-\operatorname{erf}\left(v_{\min }, \mu_{v_{2}}, \sigma_{v_{2}}\right)},
\end{aligned}
$$

where $v_{d}$ is the velocity difference that is always greater than 0 . Further we have

$$
\begin{aligned}
& \frac{S_{\text {position }}-R}{v_{d}} \leq t \leq \Psi \\
& S_{\text {position }} \leq v_{d} \Psi+R \\
& S_{\text {blind }} \leq v_{d} \Psi+R .
\end{aligned}
$$

t last, we consider the medium traffic flow density situation. As shown in Figure 4, there is a cluster formed by a few vehicles in the blind area, supposing they keep their speed until they reach RSU radio coverage areas. Here, with respect to the cluster size, we need focus on the probability that the cluster receives the warning message. We have the equation below

$$
\begin{aligned}
& P S_{\text {position }} \leq S_{\text {threshold }}=\frac{S_{\text {threshold }}}{S_{\text {blind }}} \\
& S_{\text {threshold }}=S_{\text {blind }}+2 R+\Psi v_{\text {cluster }}-\psi R-v_{\text {cluster }} t_{\text {rsu }}
\end{aligned}
$$

\section{B. Optimal deployment method of RSUs}

As the time delay is a random variable, we take the expectation of the variable when calculating the results. The expectation of $t_{r s u}$ is

$$
\begin{aligned}
E\left\{t_{r s u}\right\} & =\int_{0}^{t_{\text {rsumax }}} t f\left(t_{r s u}\right) d t \\
& =\frac{1}{S_{\text {blind }}} \int_{0}^{t_{\text {rsumax }}} t\left(\int_{v_{\text {min }}}^{v_{\text {max }}} v f(v) d v\right) d t \\
& =\frac{1}{S_{\text {blind }}} \mu_{v} \int_{0}^{t_{\text {rsumax }}} t d t \\
& =\frac{S_{\text {blind }} \mu_{v}}{2 v_{\text {min }}^{2}} .
\end{aligned}
$$

We take expectation on both sides of inequality 9 and substituting 3. We have

$$
N \geq \frac{L}{v_{\min }\left(\frac{2 R}{\mu_{v}}-\Psi\right)+2 R}-1 .
$$

\section{EXPERIMENTS}

Time delay is the critical index in high speed vehicular environment. Only the prompt time delay may help the following vehicle, which could have enough time to decelerate. Some simulation parameters are shown in Table $\mathbf{I}$.

The expected number of RSUs along the highway $(100 \mathrm{~km})$ calculated with different average and standard variance of speed under Figure 3 scenario are shown in Table II.
TABLE I The value of parameters used in the simulation

\begin{tabular}{cc}
\hline Parameter & Value \\
\hline Safety time $\Psi$ & $15 \mathrm{~s}$ \\
Cluster size coefficient $\psi$ & 2 \\
Transmission range $R$ & $300 \mathrm{~m}$ \\
Maximum speed $v_{\max }$ & $120 \mathrm{~km} / \mathrm{h}$ \\
Minimum speed $v_{\min }$ & $60 \mathrm{~km} / \mathrm{h}$ \\
\hline
\end{tabular}

TABLE II Different average and standard variance values

\begin{tabular}{cccc}
\hline$\mu_{v}(\mathrm{~km} / \mathrm{h})$ & $\sigma_{v}(\mathrm{~km} / \mathrm{h})$ & $E[N]$ & $\rho=\frac{E[N]+1}{L}(\mathrm{veh} / \mathrm{km})$ \\
\hline 90 & 20 & 85 & 0.86 \\
90 & 25 & 76 & 0.77 \\
90 & 27 & 73 & 0.74 \\
90 & 30 & 68 & 0.69 \\
80 & 27 & 68 & 0.69 \\
85 & 27 & 71 & 0.72 \\
100 & 27 & 72 & 0.73 \\
\hline
\end{tabular}

According to the simulation results, the standard variance of speed has an impact on the number of RSUs. When the standard variance is bigger, the expected number of RSUs is smaller. Figure 10 shows the other conditions that occurrence probability is lower than the above scenario. When highway length is $100 \mathrm{~km}$, the total average speed, the standard variance and the expected number of RSUs is $90 \mathrm{~km} / \mathrm{h}, 27 \mathrm{~km} / \mathrm{h}$ and 28 respectively. We compare with the results in Table II, the expected number of RSUs is 73. From the comparisons above we can learn that different models fluctuate the results. Based on the simple principle we all know, the bigger number of RSUs, the smaller the blind areas. And we should take the result from higher occurrence probability scenarios. So we assume the bigger number of RSUs is more reliable. Under medium TFD situation, when $\mathrm{N}$ is bigger than 106, we assume the cluster can $100 \%$ receive the warning message generated by RSU and have enough distance to decelerate. According to (3), the distance of blind area is $335 \mathrm{~m}$, which is smaller than the biggest cluster size, which means the cluster can make temporary coverage for the blind area. Furthermore, we use NS2 simulating the scenario described in Figure 3. The parameters are shown in Table III. The simulation time, highway length, communication range, average vehicle velocity, maximum vehicle velocity and minimum vehicle velocity is $100 \mathrm{~s}, 100 \mathrm{~km}, 250 \mathrm{~m}, 90 \mathrm{~km} / \mathrm{h}, 120 \mathrm{~km} / \mathrm{h}, 60 \mathrm{~km} / \mathrm{h}$ respectively.

TABLE III NS2 simulation parameters

\begin{tabular}{cc}
\hline Variables & Value \\
\hline Propagation & TwoRayGround \\
MAC & 802.11 \\
Queue & DropTail/PriQueue \\
Antenna & OmniAntenna \\
Routing protocol & DSDV \\
Packet Size & 200 \\
Interval & 1 \\
\hline
\end{tabular}

\section{RESUlts AND Discussion}

Figure 6 shows the relationship between the number of RSUs and the average speed. Also we can have the probability 


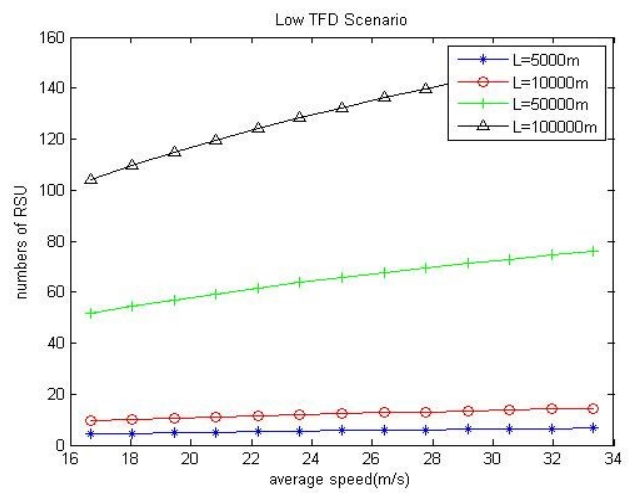

Fig. 6 Relationship between RSU number and vehicle speed.

of the RSU number with different standard deviations when the average speed is fixed, as we show in Figure 7.

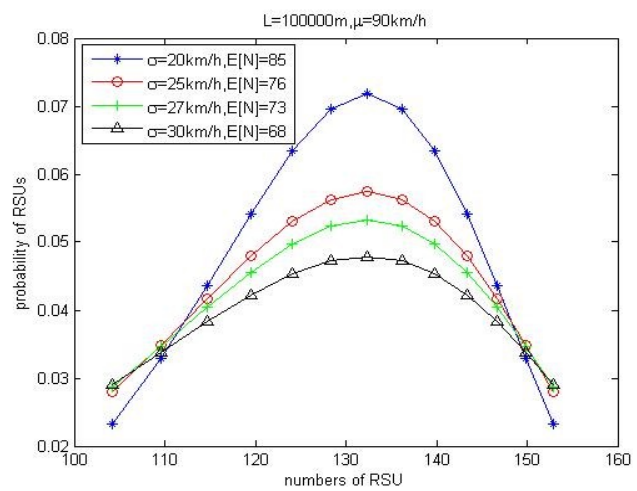

Fig. 7 Relationship between RSU number and standard deviation of vehicle speed.

In a similar way we can have the probability of the RSU number with different average speeds when the standard deviation is fixed, as we show in Figure 8.

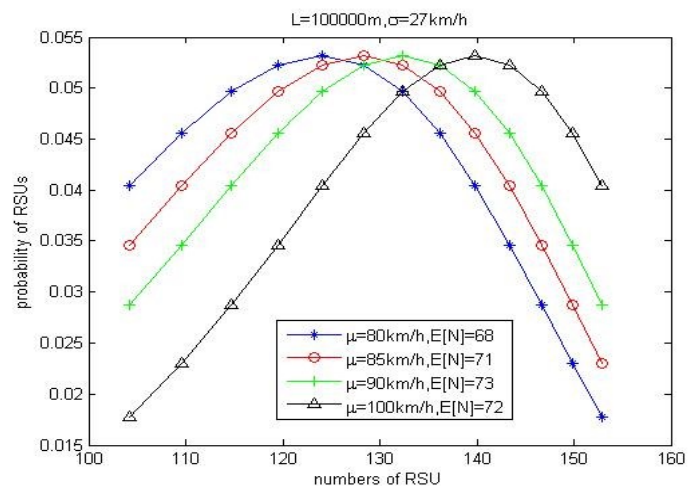

Fig. 8 Relationship between RSU number and probability.

In terms of Figure 5 situation, we take expectation on both sides of inequality (12) and substituting (3), then we have

$$
N \geq \frac{L}{\mu_{v d} \Psi+3 R}-1 .
$$

The relationship between the average velocity difference and the number of RSUs is shown in Figure 9.

For a two lane highway, there should always be a velocity difference between the express lane and the slow lane. So

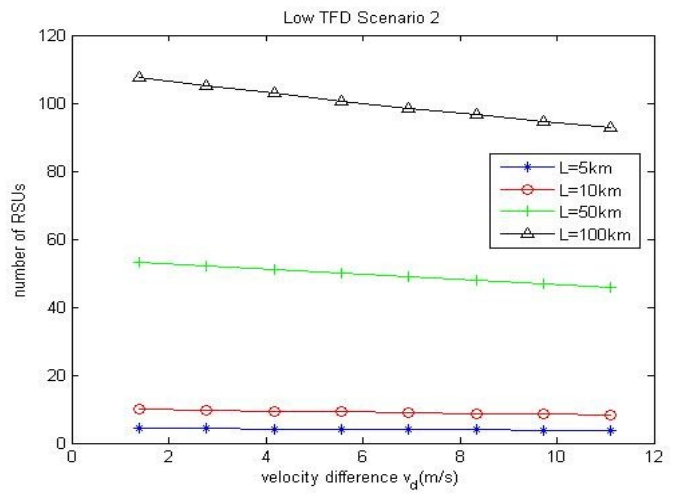

Fig. 9 Relationship between velocity difference and RSU number.

we can have the relationship between RSU numbers and the probability of the velocity difference with fixed average speed and standard deviation shown in Figure 10.

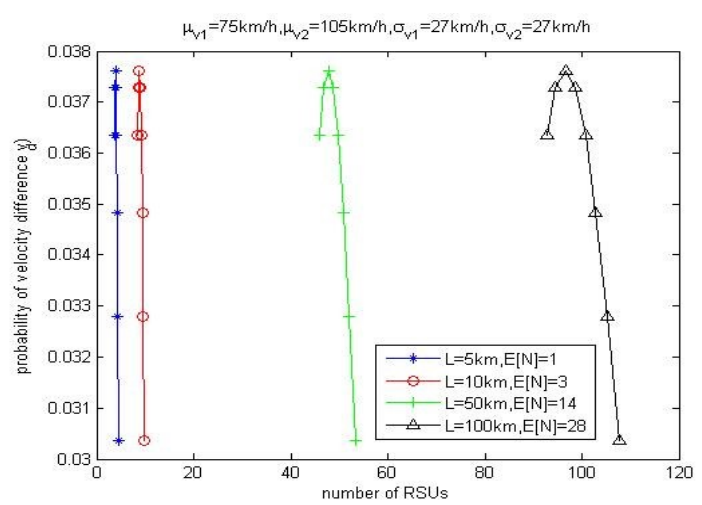

Fig. 10 Relationship between velocity difference and RSU number on two lane highway.

Finally we compute the probability of cluster vehicle receiving the warning message with (13),(14),(15). For the sake of simplicity, we assume the cluster velocity is the average vehicle speed. The probability close to zero is $\mathrm{N}$ equal to 27 when $\mathrm{L}=100 \mathrm{~km}$. In addition we compare the blind area with the cluster size. To satisfy blind area longer than cluster size, $\mathrm{N}$ should be smaller than 82 . When the probability is near to $1, \mathrm{~N}$ is equal to 106 , so we take $\mathrm{N}$ from 27 to 106. The relationship of cluster velocity, numbers of RSUs and the probability of cluster vehicle receiving the warning message is shown in Figure 11.

We test the packet loss rate of the vehicle that is in the nearest blind area to the accident site like Figure 3, and the results are shown in Figure 12. The results illustrate once the vehicle is nearer to the RSU coverage marginal, the packet loss rate is high, because the warning message is still not generated by the RSU yet, the vehicle is driving forward. Anyway, the vehicle still receives a part of the warning message packets. And wherever the vehicle is, it will receive the warning message although the packet loss rate is higher. Due to the features of the safety application message, once the vehicle receives message, it will help the driver being aware of the danger ahead. So the model is quite suitable to the scenarios in Figure 3.

In comparison with TAPC method [10], The findings can be 


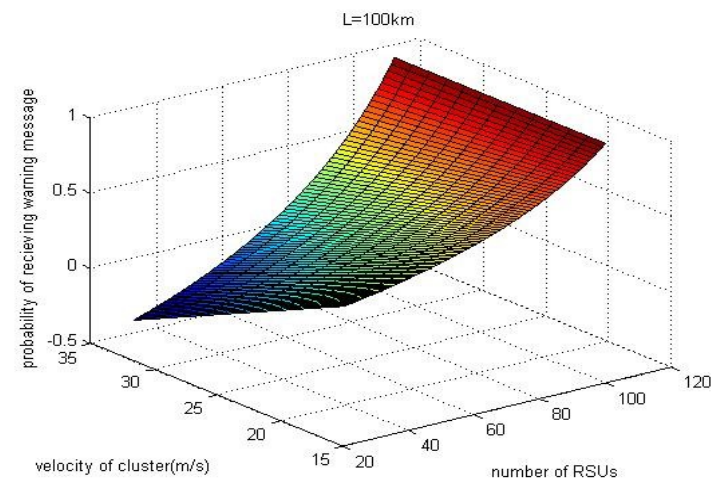

Fig. 11 Relationship between velocity, RSU number and probability of receiving message.

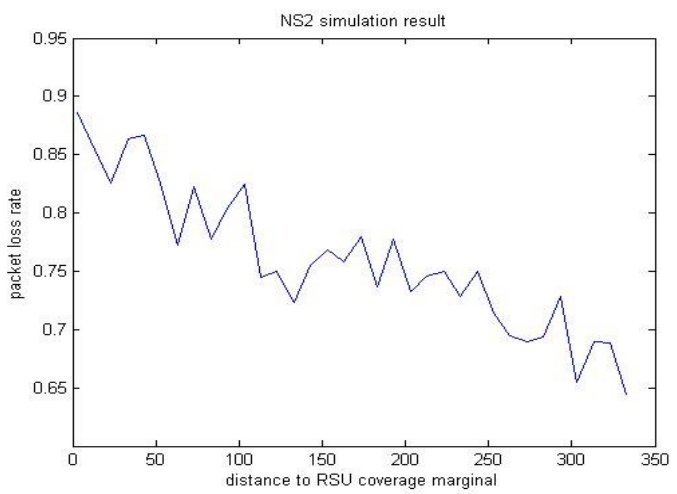

Fig. 12 Relationship between packet loss rate and distance to RSU coverage marginal.

seen in Figure 13. When the probability of receiving warning message is higher than $92 \%$, the interval between two RSUs calculated by our proposed method is bigger than TAPC method, which means our proposed method is better than TAPC method in condition of higher receiving probability. Due to the need for safety application, higher probability circumstance is our main focus. The TAPC method is calculated mainly based on power consumption, Which we do not think is necessary when receiving important safety information.

Above all, for the sake of safety application, the optimal RSU numbers along the highway should be the biggest number that is given by the three above model. In consideration of better performance, RSUs should be uniformly distributed along the highway. From a realistic point of view, gas stations, resting areas, road lamp locale and etc. will be the RSU locations, so the actual blind area range is smaller than theoretical calculation.

\section{CONCLUSiON}

In this paper, we proposed a practical model for computing the optimal RSU number based on a single and clusters of vehicle in smart IoV. Our model integrated three normal situation in the highway, and we also derived closed-form expressions for different situations, validated by extensive simulations. The proposed model and analysis provide guidelines for the design and management of smart IoV to achieve the balance of economic factors and safety application performance. In future works, one can consider extending our model to adapt more complicated road shapes.

\section{ABBREVIATIONS}

IoV: Internet of Vehicle

RSU: RoadSide Unit

ITS: Intelligent Transportation System

V2R: Vehicle-to-Roadside Unit

V2V: Vehicle-to-Vehicle

QoS: Quality of Service

VANETs: Vehicular Ad Hoc Networks

TAPC: Traffic-Aware Power Control

BIP: Binary Integer Programming

BEH: Balloon Expansion Heuristic

CDF: Cumulative Distribution Function

\section{REFERENCES}

[1] "Global Status Report on Road Safety 2013," http://www.who.int/

[2] H. Su and X. Zhang,, "Clustering-based multichannel MAC protocols for QoS provisionings over vehicular ad hoc networks," IEEE Trans. Veh. Technol., vol. 56, no. 6, pp. 3309-3323, 2007.

[3] O. Kayis and T. Acarman,, "Clustering formation for inter-vehicle communication," in Proc. of IEEE Intelligent Transportation Systems Conference, Seattle, WA, USA, Sep. 2007, pp. 636-641.

[4] Z. Wang, L. Liu, M. Zhou, and N. Ansari, "A position based clustering technique for ad hoc intervehicle communication," IEEE Trans. Syst, Man, and Cyber.,Part C: Applications and Reviews, vol. 38, no. 2, pp. 201208, 2008.

[5] C. Shea, B. Hassanabadi, and S. Valaee "Mobility-based clustering in VANETs using affinity propagation," in Proc. 2009 IEEE Global Telecommunications Conf. (GLOBECOM), 2009, pp. 16.

[6] Z. Y. Rawashdeh and S. M. Mahmud, "A novel algorithm to form stable clusters in vehicular ad hoc networks on highways," EURASIP Journal on Wireless Communications and Networking, vol. 20, no. 12, pp. 15, 2012.

[7] Liu, C., Huang, H, and Du. H. J, Optimal RSUs deployment with delay bound along highways in VANET, Journal of Combinatorial Optimization, pp. 1-15, 2016.

[8] O. Trullols, M. Fiore, and C. Casetti et al., Planning roadside infrastructure for information dissemination in intelligent transportation systems, Computer Communications, vol. 33, no.4, pp. 432442, 2010.

[9] B. Aslam, F. Amjad, and C. C. ZouOptimal roadside units placement in urban areas for vehicular networks, in Proc. IEEE Symposium on Computers and Communications(ISCC), pp. 423-429, 2012.

[10] J. Tao, L. Zhu, X. Wang, J. He, and Y. Liu RSU deployment scheme with power control for highway message propagation in VANETs, in Proc. Global Communications Conf., pp.169-174, 2015.

[11] R. Cumbal, H. Palacios, and R. Hincapie Optimum deployment of RSU for efficient communications multi-hop from vehicle to infrastructure on VANET in Proc. IEEE Colombian Conference on Communications and Computing (COLCOM), pp. 1-6, 2016.

[12] Y. Xiong, J. Ma, W. Wang, and D. Tu, RoadGate:Mobility-Centric Roadside Units deployment for Vehicular Networks International Journal of Distributed Sensor Networks, pp:547-565, 2013.

[13] H. Cheng, X. Fei, A. Boukerche, and M. Almulla, A Genetic AlgorithmBased Sparse Coverage over Urban VANETs, in Proc. IEEE International Parallel \& Distributed Processing Symposium Workshops, pp. 464-469, 2014.

[14] I. Filippini, F. Malandrino, G. Dn, M. Cesanam, C. Casetti, and I. Marsh Non-cooperative RSU deployment in Vehicular networks, in Proc. 9th Annual Conference on Wireless On-Demand Network Systems and Services (WONS), pp. 79-82, 2012.

[15] R. Massobrio, S. Bertinat, S. Nesmachnow, J. Toutouh, and E. Alba Smart placement of RSU for vehicular networks using multiobjective evolutionary algorithms, in Proc. Latin America Congress on Computational Intelligence (LA-CCI), pp. 1-6, 2015.

[16] A. Makkawi, R. Daher, and R. Rizk RSUs Placement Using Cumulative Weight Based Method for Urban and Rural Roads in Proc. 7th International Workshop on Reliable Networks Design and Modeling (RNDM), pp. 307-313, 2015. 
[17] N. Balouchzahi, M. Fathy, and A. Akbari Optimal road side units placement model based on binary integer programming for efficient traffic information advertisement and discovery in vehicular environment IET Intelligent Transport Systems, pp. 851-861, 2014.

[18] L. Xu, C. Huang, P. Li, and J. Zhu A Randomized Algorithm for Roadside Units Placement in Vehicular Ad Hoc Network in Proc. IEEE 9th International Conference on Mobile Ad-hoc and Sensor Networks, pp. 193-197, 2013.

[19] T. Wu, W. Liao and C. Chang, A Cost-Effective Strategy for Road-Side Unit Placement in Vehicular Networks IEEE Transactions on Communications, vol. 60, no. 8, pp. 2295-2303, 2012.

[20] C. B. Ancharev, "Connectivity analysis of one-dimensional vehicular ad hoc networks in fading channels," EURASIP Journal on Wireless Communications\&Networking., 2012.

\section{DeClarations}

Ethics Approval and Consent to Participate

Not applicable.

Consent for Publication

Not applicable.

Availability of Data and Materials

The datasets used for the evaluation of the algorithm are not sharing publicly. Please contact the corresponding author if neccessary.

\section{Competing Interests}

The authors declare that they have on competing interests.

\section{Funding}

This paper is supported by Heilongjiang Provincial Natural Science Foundation of China (2018023).

\section{Author's Contributions}

Honghan Zhang developed and implemented the concepts of the algorithm presented within this manuscript. The author read and approved the final manuscript.

\section{Acknowledgments}

The author would like to thank the anonymous reviewers for their valuable suggestions.

\section{Author's Information}

Honghan Zhang has a Ph. D in Control Theory and Control Engineering, specialises in random signal processing and artificial intelligence, and is currently an Associate Professor with college of intelligent system science and engineering, Harbin Engineering University, China.

\section{Author's Affiliations}

Harbin Engineering University, College of Intelligent System Science and Engineering

Honghan Zhang

\section{Corresponding author}

Correspondence to Honghan Zhang. 